\title{
A vitamina $D$ e desempenho imunológico: uma perspectiva dentro da esclerose múltipla
}

The vitamin D and immunological performance: a perspective within multiple sclerosis

La vitamina D e rendimento imunológico: uma perspectiva dentro de la eslerosis múltiple

Raquel Araújo Nogueira

ORCID: https://orcid.org/0000-0001-8535-6847 Instituto de Educação Superior Vale do Parnaíba, Brasil

E-mail: raquelaraujo852@gmail.com

Célia Barros de Sousa Marques

ORCID: https://orcid.org/0000-0001-9309-1893 Instituto de Educação Superior Vale do Parnaíba, Brasil E-mail: celiamarques_@outlook.com

Ana Clara Correia Gomes

ORCID: https://orcid.org/0000-0003-2463-0720 Instituto de Educação Superior Vale do Parnaíba, Brasil

E-mail: clarinhacorreiaanaclaracorreia@hotmail.com

Leiz Maria Costa Véras

ORCID: https://orcid.org/0000-0003-3422-2878 Instituto de Educação Superior Vale do Parnaíba, Brasil E-mail: leiz.vera@iesvap.edu.br

Ariellen Martins Guerra

ORCID: https://orcid.org/0000-0001-6201-2806 Instituto de Educação Superior Vale do Parnaíba, Brasil E-mail: ariellenmartins1@hormail.com

Guilherme Araújo da Silva

ORCID: https://orcid.org/0000-0001-5579-4459 Instituto de Educação Superior Vale do Parnaíba, Brasil E-mail: guilherme11araujo@gmail.com

Antônio Victor de Oliveira Machado

ORCID: https://orcid.org/0000-0003-0016-5959 Instituto de Educação Superior Vale do Parnaíba, Brasil E-mail: victor2711@live.com

Maria Clara Nolasco Alves Barbosa

ORCID: http://orcid.org/ 0000-0002-1275-2678 Instituto de Educação Superior Vale do Parnaíba, Brasil E-mail: nolascomariab@gmail.com

Francisco Hildebrando Moreira de Oliveira Filho

ORCID: https://orcid.org/0000-0002-8362-4374 Instituto de Educação Superior Vale do Parnaíba, Brasil E-mail: bambam.igt@gmail.com

Maria das Graças Resende da Silva Neta

ORCID: https://orcid.org/0000-0002-0945-1751 Instituto de Educação Superior Vale do Parnaíba, Brasil E-mail: gracinharesende@outlook.com

Lucas Benjamim Pereira Farias

ORCID: https://orcid.org/0000-0002-9133-1475 Instituto de Educação Superior Vale do Parnaíba, Brasil

E-mail: lucasbenjamim35@hotmail.com

Francisco de Nojosa Costa Neto

ORCID: https://orcid.org/0000-0001-8721-5087 Instituto de Educação Superior Vale do Parnaíba, Brasil E-mail: netotoc99@hotmail.com

David Danisio Silva de Freitas

ORCID: https://orcid.org/0000-0003-4003-1971 Instituto de Educação Superior Vale do Parnaíba, Brasil E-mail: fullbringboy@gmail.com

Alyne de Araújo Paiva

ORCID: https://orcid.org/0000-0002-1950-0887 Instituto de Educação Superior Vale do Parnaíba, Brasil E-mail: alynepaivaa@icloud.com 


\begin{abstract}
Resumo
Introdução: A esclerose múltipla (EM) é uma doença autoimune, crônica e degenerativa no qual gera inflamações em regiões cerebrais e da medula espinhal. A doença pode acometer qualquer região do sistema nervoso central cursando com sintomas variados. Suas causas são multifatoriais, relacionando-se com componente genético, comportamentais, ambientais e imunológicos mediados pela bioquímica da vitamina D. A vitamina D possui função na mediação imunológica no processo anti-inflamatório, no que tange reduzir os níveis de citocinas inflamatórias e potencializar as anti-inflamatórias no processo de suplementação de seis meses. Objetivo: Analisar as vantagens do uso de vitamina D em pacientes com esclerose múltipla. Metodologia: Trata-se de uma revisão integrativa da literatura. Realizou-se levantamento bibliográfico de artigos nas bases de dados: PubMed, SciELO e Medline, no período de 2010 a 2020, com o uso dos descritores: "Vitamina D"; "Esclerose Múltipla" e "Sistema Imune". Os critérios de inclusão abrangeram artigos em língua inglesa e portuguesa limitados ao período de 10 anos, disponíveis na íntegra. Excluíram-se teses, revisões teóricas, capítulos de livros e artigos duplicados. Resultados: No total, nove estudos foram analisados na presente revisão. Verificou-se na literatura, que a vitamina D desempenha um papel importante no processo de reabilitação dos pacientes com esclerose múltipla, destacando sua ação na inibição dos mediadores inflamatórios após influência da suplementação continua com vitamina D. Conclusão: Portanto, que o uso de Vitamina D mostra bons resultados no tratamento e recuperação dos portadores da doença esclerose múltipla. No entanto, é necessário realizar estudos mais detalhados em relação às doses e possíveis efeitos deletérios.
\end{abstract}

Palavras-chave: Vitamina D; Sistema imune; Esclerose múltipla.

\begin{abstract}
Introduction: Multiple sclerosis (MS) is a chronic, degenerative, autoimmune disease that generates inflammation in brain and spinal cord regions. The disease can affect any region of the central nervous system, with a variety of symptoms. Its causes are multifactorial, being related to genetic, behavioral, environmental, and immunological components mediated by vitamin D biochemistry. Vitamin D has a function in immune mediation in the antiinflammatory process, in what concerns reducing the levels of inflammatory cytokines and potentiating the antiinflammatory ones in the process of six months supplementation. Objective: To analyze the advantages of the use of vitamin D in patients with multiple sclerosis. Methodology: This is an integrative literature review. A bibliographic search of articles in the PubMed, SciELO and Medline databases was carried out from 2010 to 2020, using the descriptors: "Vitamin D", "Multiple Sclerosis" and "Immune System". Inclusion criteria included articles in English and Portuguese limited to a period of 10 years, available in full. Theses, theoretical reviews, book chapters and duplicate articles were excluded. Results: A total of nine studies were analyzed in this review. It was found in the literature, that vitamin D plays an important role in the rehabilitation process of patients with multiple sclerosis, highlighting its action in the inhibition of inflammatory mediators after the influence of continuous supplementation with vitamin D. Conclusion: Therefore, that the use of Vitamin D shows good results in the treatment and recovery of multiple sclerosis disease sufferers. However, more detailed studies are needed regarding doses and possible deleterious effects.
\end{abstract}

Keywords: Vitamin D; Immune system; Multiple sclerosis.

\title{
Resumen
}

Introducción: La esclerosis múltiple (EM) es una enfermedad autoinmune, crónica y degenerativa que genera inflamación en regiones del cerebro y la médula espinal. La enfermedad puede afectar a cualquier región del sistema nervioso central con diversos síntomas. Sus causas son multifactoriales, relacionadas con componentes genéticos, conductuales, ambientales e inmunológicos mediados por la bioquímica de la vitamina D. La vitamina $\mathrm{D}$ tiene una función en la mediación inmunológica en el proceso antiinflamatorio, lo que permite reducir las tasas de citocinas inflamatorias y potenciar las antiinflamatorias en el proceso de suplementación de seis meses. Objetivo: Analizar las ventajas del uso de la vitamina $\mathrm{D}$ en pacientes con esclerosis múltiple. Metodología: Se trata de una revisión integradora de la literatura. Se realizó un estudio bibliográfico de artículos en las siguientes bases de datos: PubMed, SciELO y Medline, desde 2010 hasta 2020, utilizando los descriptores: "Vitamina D"; "Esclerosis múltiple" y "Sistema inmunológico". Los criterios de inclusión incluyeron artículos en inglés y portugués limitados a un período de 10 años, disponibles en su totalidad. Se excluyeron las tesis, las revisiones teóricas, los capítulos de libros y los artículos duplicados. Resultados: En esta revisión se analizaron un total de nueve estudios. Se ha comprobado en la literatura que la vitamina D desempeña un papel importante en el proceso de rehabilitación de los pacientes con esclerosis múltiple, destacando su acción en la inhibición de los mediadores inflamatorios tras la influencia del suplemento continuo de vitamina D. Conclusión: Por lo tanto, que el uso de la vitamina D muestra buenos resultados en el tratamiento y la recuperación de los portadores de la enfermedad de la esclerosis múltiple. Por lo tanto, es necesario realizar estudios más detallados en relación con las dosis y los posibles efectos de la eliminación.

Palabras clave: Vitamina D; Sistema inmunológico; Esclerosis múltiple. 


\section{Introdução}

A esclerose múltipla (EM) é uma doença crônica, heterogênea, progressiva, neurodegenerativa, autoimune e desmielinizante, sendo a mais comum do sistema nervoso central (SNC) o qual é constituído pelo encéfalo e medula espinhal. Dessa maneira, a EM é caracterizada pela destruição da bainha de mielina uma capa de tecido adiposo presente na substância branca que recobre o axônio e aumenta a velocidade de propagação do impulso elétrico (Silva, 2014). Consequentemente a degradação da bainha, o indivíduo apresentará manifestações e curso clínico muito variável como fraqueza, diplopia, ataxia, vertigem, parestesia, dificuldades de locomoção, podendo também apresentar outros sintomas como rigidez muscular, déficits cognitivos e dificuldades para realização da micção e da defecação (Bertotti, 2011).

Essa doença pode se apresentar sob 4 formas clínicas: síndrome clínica isolada; recorrente-remitente; primariamente progressiva e secundariamente progressiva (Campos, 2015). A síndrome clínica isolada é a forma que ocorre com menor frequência, sendo definida pelo primeiro episódio clínico sugestivo de um quadro inflamatório desmielinizante com deterioração progressiva desde o início dos sintomas, tornando-se necessário avaliar outras etiologias mimetizantes para o diagnóstico, pois $20 \%$ dos pacientes não convertem para a EM (Errante, 2016).

O tipo mais comum de EM é a remitente-recorrente caracterizada pela presença de áreas focais de inflamação e desmielinização com períodos de crise ocasionando intensificação dos sintomas e períodos de estabilidade clínica. As duas outras formas de apresentações clínicas da doença são a primariamente progressiva na qual é descrita pelo processo de degeneração gradual sem presença evidente de surtos, com danos irreparáveis e a secundariamente progressiva é representada pelo início reversível de uma disfunção neurológica com atrofia cerebral sem a presença de um número grande de crises (Errante, 2016).

Por ser caracterizada como uma doença heterogênea tem a influência de vários fatores genéticos e ambientais. Os fatores de origem ambientais mostram que a prevalência de EM possui intrínseca relação com a exposição solar na qual se concentram principalmente em países de baixa latitude (Brum, 2014). Consequentemente, os habitantes de países próximo a linha do Equador tem maior captação de raios ultravioletas pela pele, com aumento da vitamina D no organismo que diminuindo, assim, a probabilidade de desenvolver a doença (Errante, 2016).

Outro fator ambiental envolvido a predisposição dessa enfermidade é o tabagismo, pois de acordo com pesquisas, observa-se que os fumantes têm o risco de 1,3 a 1,8 vezes maior de serem acometidos do que os não fumantes. Relacionado a genética nota-se que mulheres (3:1), caucasianos, adultos de 20 a 40 anos de idade e moradores do norte europeu tem maior tendência a adquirir essa enfermidade (Errante, 2016).

Esse trabalho tem como objetivo enfatizar sobre a importância de avaliar a relação da vitamina $D$ em pacientes com esclerose múltipla, buscando em base de dados artigos relacionados a essa temática. Além disso, foi objetivado entendermos melhor a metabolização da Vitamina D no organismo, sua atuação na imunidade inata e adquirida, as formas de adquirir Vitamina D e como a influência climática afeta na dosagem orgânica de Vitamina D e a prevalência de Esclerose Múltipla.

\section{A vitamina $D$}

Há mais de quatro décadas estuda-se a importância da vitamina $\mathrm{D}$, sendo descoberta sua função na regulação da imunidade inata e adquirida (Marques,2010). Visto isso, foi pensado em como essa sua função poderia colaborar na melhora de pacientes com doenças autoimunes, onde é possível encontrar o sistema imunológico debilitado. Dessa forma, foi possível observar o aumento de citocinas e interleucinas anti-inflamatórias além da diminuição do Fator de Necrose Tumoral (FNT), interferon e linfócitos $\mathrm{T}$ quando submetidos a doses de vitaminas $\mathrm{D}$ diárias, tendo uma significativa melhora do quadro do paciente (Alves,2014).

A vitamina D é considerada um hormônio esteroide, anti-inflamatório precursor do colesterol e com alto potencial 
antioxidante (Marques,2010), devido as suas propriedades metabólicas e sua ativação de mais de 200 genes funcionais no organismo. De acordo com a Sociedade Brasileira de Pediatria - SBP (2016), a Vitamina D é subdividida em dois tipos: vitamina D2 e vitamina D3. A vitamina D2 ou ergocalciferol é encontrada pelo consumo de alimentos de origem vegetal como cogumelos. A vitamina D3 ou colecalciferol é obtida tanto pela dieta animal de peixes (salmão, atum e bagre), óleo do fígado do bacalhau, gema de ovo, queijo, leite e fígado quanto pela produção cutânea na epiderme (Catarino,2016).

Vale ressaltar que a dieta oral suprime apenas $20 \%$ da capacidade diária, sendo os outros $80 \%$ a principal fonte de obtenção da vitamina $\mathrm{D}$ através da exposição aos raios solares que ao serem captados pelo tecido corporal, iniciam a produção endógena de calcitriol que se dá pela metabolização da vitamina D no organismo (Galvão,2013).

Tal metabolização inicia-se no momento que os raios UVB penetram na pele e são absorvidos pelas células epiteliais. A partir dessa exposição, a molécula 7-dehidrocolesterol é convertida em pré- vitamina D3 termoinstável, ou seja, sofrerá uma isomerização dependente da temperatura e iniciará o processo de sintetização do colecalciferol (D3), forma biologicamente inativa (Araújo,2018). A vitamina D2 e o pré-vitamina D3 entram na circulação sanguínea absorvida pelo intestino através da dieta ou sintetizada cutaneamente transformando-se em ergosterol e colecalciferol, respectivamente. Logo após ligam-se a proteína específica da vitamina D (DBP), na qual é produzida pelo fígado (Castro,2011). Depois disso, são transportadas para o fígado onde sofrerão hidroxilação no carbono 25 pela enzima 25-hidroxilase, sendo o ergosterol transformado em 25hidroxiergocalciferol enquanto o colecalciferol dá origem ao 25-hidroxicolecalciferol, sendo suas junções chamada de 25hidroxivitamina D ou calcidiol (Maeda,2014). Esta é a forma metabólica em maior abundância no organismo e com maior tempo de meia vida, que fica circulante por até 3 semanas indicando a quantidade de vitamina D no corpo.

Em seguida, a 25-hidroxivitamina D é absorvida no rim, mais precisamente no túbulo contorcido proximal onde sofre um processo de hidroxilação pela enzima 1 alfa-hidroxilase (CYP27B1) sob ação do hormônio paratireoide (Castro,2011) e é convertida em calcitriol, cuja molécula é 1,25-dihidroxicolecalciferol [(1,25(OH)2D3)] ou 1,25-desidroxivitamina D, sendo a forma ativa da vitamina (Maeda,2014). Por fim, as moléculas ativas são levadas para as células de vários órgãos pela DBP, ligando- se aos receptores de vitamina D (VDR) e fazendo sua participação nos processos metabólicos (Catarino,2016).

Como citado anterior, o efeito da vitamina D no sistema imunológico é transformado em um aumento da imunidade inata, que está relacionada à regulação da imunidade adquirida (Amado,2015). Tal relação é estabelecia devido a seus mecanismos de combate a antígenos e o seu aumento da imunidade. Tal aumento se dá por meio de ativação, transcrição e diferenciação de células imunitárias e complexos metabólicos. Na imunidade inata, a vitamina D atua na diferenciação de monócitos, que são células de defesa imaturas, em macrófagos, que são glóbulos brancos capazes de fagocitar invasores, células danificadas e envelhecidas, além de inibir a diferenciação dos monócitos em células dendríticas (CD) (Biasebetti, 2018), que são as principais células apresentadoras de antígenos (APCs) e ao serem expostas a vitamina D reduz-se o complexo de histocompatibilidade II, suprimindo a apresentação de antígenos no organismo com menor secreção de IL-2 e aumento da IL10. assim, ocorrendo a diferenciação de linfócitos th2 e aumentando a tolerância imune. Ademais, o calcitriol tem um papel importantíssimo na transcrição da catelicidina e defensina, pois tais peptídeos anti-microbianos (AMPs) após sofrerem o processo de transcrição apresentam atividades imunomoduladoras inibitórias de bactérias, fungos e vírus (Marques, 2010).

$\mathrm{Na}$ imunidade adquirida, estão relacionadas as células TCD4+ e TCD8+ do sistema imune com a vitamina D (Siqueira, 2016). Tem se a influência da molécula $1,25(\mathrm{OH}) 2 \mathrm{D}$ por meio da proliferação diminuída dos linfócitos, principalmente das células Th helper, promovendo sua conversão para Th2. Ademais, a conversão da exposição solar em vitamina D juntamente com as células de defesa TCD4+ possibilitam a inibição de citocinas pró-inflamatórias Th1, Th9, Th22, fator de necrose tumoral (FNT) e estimulam a produção de citocinas anti-inflamatórias Th2, como IL-3, IL-4, IL-5 e IL-10 (Aranow, 2011).

Além disso, estudos apontam que o calcitriol cuja molécula é 1,25-dihidroxicolecalciferol na sua forma ativa, diminuem a produção de IL-17, favorecendo o desvio para a via Th2 (Castro, 2011), diminuindo a probabilidade de processos inflamatórios 
e desenvolvimento de doenças autoimunes. Por fim, as células TCD8+ citotóxicas tem muitos receptores de vitamina D (RVD) o que favorece a regulação de citocinas e da proliferação dessas células.

Dessa forma, observa-se que a esclerose múltipla está relacionada com a proliferação do FNT, produção de citocinas pró-inflamatórias, aumento das interleucinas inflamatórias: 2,6,9, 17 e 22 e indução da via Th1, promovendo a patogênese da EM (Souza, 2010). Contudo, o papel a vitamina D está em aumentar citocinas anti-inflamatórias: 3,4,5 e 10, diminuir interferongama (INFy) e FNT, inibir a diferenciação e transcrição de células imunomoduladoras parácrinas e desvia as células linfocitárias para a via Th2, promovendo a regulação do sistema imunológico e contribuindo para a redução do risco de desenvolvimento da esclerose múltipla (Brito, 2017).

\section{Metodologia}

O estudo trata-se de uma revisão integrativa da literatura, que segundo que segundo Souza, Silva e Carvalho (2010), a revisão integrativa da literatura busca tomar conhecimento e fazer uma avaliação das colaborações científicas acerca de um determinado assunto, tema ou problemática. Foi utilizada a estratégia PICo, que representa um acrômio para População/Paciente (adulto com esclerose múltipla), Interesse (familiares com pacientes de esclerose múltipla), Contexto (ambulatórios), para a definição da questão de investigação e dos critérios de inclusão e exclusão. Usou-se da pergunta norteadora: "Qual a eficácia da vitamina D no tratamento da esclerose múltipla?"

Para a pesquisa, foram analisados artigos indexados nas bases de dados: Pubmed, Scielo e Medline, utilizando os descritores: "VITAMINA D", "SISTEMA IMUNE” e "ESCLEROSE MÚLTIPLA. Todos os descritores aplicados para a pesquisa foram confirmados no Descritores em Ciências da Saúde (DeCS), edição 2020. Associada a essa busca, foi correlacionado o uso do operado booleano "AND" aos termos "VITAMINA D" e "ESCLEROSE MÚLTIPLA".

Uma combinação de especificações foi estabelecida para o estudo, sendo firmado como critérios de inclusão artigos completos publicados entre 2010 e 2020 nos idiomas ingleses ou português, disponíveis na integra gratuitamente. Já os critérios de exclusão foram pautados em artigos duplicados, teses, revisões teóricas, capítulos de livros, artigos fora dos idiomas estabelecidos e artigos que não respondiam a temática conjuntamente.

Após a seleção dos artigos houve a construção de um banco de dados de integração das informações dos artigos pesquisados, no qual foram organizados os estudos em quadros, utilizando-se do programa Microsoft Word e Excel 2010. Nesse interim, a presente revisão foi realizada em três etapas. A princípio, as publicações analisadas foram selecionadas de acordo com o título, sendo escolhidos apenas aqueles que estavam associados a vitamina D. A segunda etapa envolveu a leitura de resumos e a seleção de estudos que possui relevância com a vitamina D. Portanto a etapa final contempla a leitura completa de artigos selecionadas na integra que comprovam os potenciais efeitos dos tratamentos através do uso de vitamina D.

\section{Resultados e Discussão}

De acordo com a estratégia definida, a busca bibliográfica nas bases de dados online somou o total de 192 artigos resultando na seleção de 9 artigos científicos apresentados no Quadro 1, conforme os critérios de inclusão e exclusão citados na metodologia, que foram utilizados para discussão e consideração finais deste estudo. 
Quadro 1: Descrição dos Artigos selecionados segundo: Título do artigo, ano, autor e resultados alcançados.

\begin{tabular}{|c|c|c|c|}
\hline Título do artigo & $\begin{array}{l}\text { Ano do } \\
\text { artigo }\end{array}$ & Autor & Resultados alcançados \\
\hline $\begin{array}{l}\text { A importância dos } \\
\text { níveis de vitamina D } \\
\text { nas doenças } \\
\text { autoimunes }\end{array}$ & 2010 & $\begin{array}{l}\text { Cláudia Diniz } \\
\text { Lopes Marques }\end{array}$ & $\begin{array}{l}\text { Segundo o estudo pesquisado foi demonstrado que a dosagem de vitamina D está } \\
\text { relacionada tanto a diminuição das taxas de recidiva quanto a prevenção do seu } \\
\text { surgimento. Na população branca o risco foi menor (em até } 40 \% \text { ) nos que } \\
\text { obtiveram alta ingesta de colecalciferol, não sendo detectado esse risco menor em } \\
\text { população negra e hispânica. Além disso, foi constatado que a dosagem de } \\
\text { vitamina D atuou prevenindo o início de encefalite autoimune alérgica e } \\
\text { lentificando também sua progressão, sendo essa uma doença que tem relação } \\
\text { fisiopatológica com a EM. }\end{array}$ \\
\hline $\begin{array}{l}\text { Vitamina D- uma } \\
\text { promessa } \\
\text { esclerose múltipla }\end{array}$ & 2013 & $\begin{array}{l}\text { Fernanda M. } \\
\text { Gomes. } \\
\text { Joana F. Almeida }\end{array}$ & $\begin{array}{l}\text { De acordo com esse artigo observa-se que não há evidencia suficiente que } \\
\text { comprove a eficácia do uso da vitamina } D \text { no tratamento da esclerose múltipla. }\end{array}$ \\
\hline $\begin{array}{l}\text { Suplementação } \mathrm{e} \\
\text { uso terapêutico de } \\
\text { vitamina D nos } \\
\text { pacientes com } \\
\text { esclerose múltipla }\end{array}$ & 2014 & $\begin{array}{l}\text { Doralina } \\
\text { Guimarães Brum }\end{array}$ & $\begin{array}{l}\text { Os estudos demonstraram que os paciente não possuem evidências científica } \\
\text { significativas nos parâmetros clínicos da EM mesmo após realizarem } \\
\text { suplementação de Vitamina D. Dessa forma, através do uso de vitamina D os } \\
\text { mesmos não apresentam nenhuma diferença significativa nos parâmetros da } \\
\text { atividade da doença. }\end{array}$ \\
\hline $\begin{array}{l}\text { Multiple sclerosis } \\
\text { patients have a } \\
\text { diminished } \\
\text { serologic response } \\
\text { to vitamin D } \\
\text { supplementation } \\
\text { compared to healthy } \\
\text { controls }\end{array}$ & 2016 & Pavan Bhargava & $\begin{array}{l}\text { De acordo com este estudo, foram avaliados pacientes com EM e indivíduos } \\
\text { saudáveis, verificando assim se os mesmos possuem mudança semelhante com } \\
\text { relação aos níveis de vitamina D após dose fixa de suplementação de vitamina D3 } \\
\text { Dessa forma, após a suplementação ocorreu um aumento significativo nos níveis } \\
\text { de vitamina D em ambos indivíduos. }\end{array}$ \\
\hline $\begin{array}{l}\text { Safety and } \\
\text { immunologic effects } \\
\text { of high-vs low-dose } \\
\text { cholecalciferol in } \\
\text { multiple sclerosis }\end{array}$ & 2016 & $\begin{array}{l}\text { Elias S Sotirchos } \\
\text { Pavan Bhargava } \\
\text { Christopher } \\
\text { Eckstein } \\
\text { Keith Van Haren } \\
\text { Moira Baynes }\end{array}$ & $\begin{array}{l}\text { Nesse estudo, foram observados e caracterizados os efeitos imunológicos da } \\
\text { suplementação de baixas e altas doses de colecalciferol em pacientes com EM } \\
\text { Dessa maneira, quando aconteceu a suplementação com altas doses de } \\
\text { colecalciferol houve um aumento significativo diante os níveis séricos de vitamina } \\
\text { D3, quando se compara com suplementação com baixas doses, desempenhando a } \\
\text { também função antioxidante e imunomodulador em pacientes com EM. Essa } \\
\text { suplementação com altas doses de colecalciferol, ocasionou também diminuição } \\
\text { na proporção de células T CD41IL-171 em pacientes com EM importante diante a } \\
\text { terapêutica de utilização de vitamina D na EM. }\end{array}$ \\
\hline $\begin{array}{l}\text { Vitamin } \\
\text { supplementation } \\
\text { reduces relapse rate } \\
\text { in } \quad \text { relapsing- } \\
\text { remitting multiple } \\
\text { sclerosis patients } \\
\text { treated } \\
\text { natalizumab }\end{array}$ & 2016 & $\begin{array}{l}\text { Julie Hejgaard } \\
\text { Laursen }\end{array}$ & $\begin{array}{l}\text { Estudos realizados para analisar os efeitos da indicação de suplementação de } \\
\text { vitamina } \mathrm{D} 3 \text { em relação aos níveis de } 25(\mathrm{OH}) \mathrm{D} \text { em pacientes com EM remitente- } \\
\text { recorrente e os achados clínicos associados as mudanças nos níveis de } 25(\mathrm{OH}) \mathrm{D} \\
\text { A análise desse estudo demonstrou que a simples recomendação e a utilização de } \\
\text { Vitamina D para pacientes com EM se associam com aumento significativo dos } \\
\text { níveis de } 25(\mathrm{OH}) \mathrm{D} \text {, ocorrendo assim uma queda na quantidade de recorrência } \\
\text { anual da EM. }\end{array}$ \\
\hline $\begin{array}{l}\text { Anti-inflammatory } \\
\text { nutritional } \\
\text { intervention in } \\
\text { patients with } \\
\text { relapsing-remitting } \\
\text { and primary- } \\
\text { progressive multiple } \\
\text { sclerosis: A pilot } \\
\text { study }\end{array}$ & 2016 & iccio & $\begin{array}{l}\text { Nesse estudo, foi realizado uma análise do estado infamatório e do bem-estar em } \\
\text { pessoas com EM isso de acordo com a avaliação da intervenção nutricional. Os } \\
\text { pacientes foram avaliados após } 3 \text { meses e } 6 \text { meses de suplementação de vitamina } \\
\text { D e intervenção nutricional, inicialmente após a administração de vitamina D os } \\
\text { pacientes não apresentaram melhoras, porém após } 3 \text { meses os ácidos graxos poli- } \\
\text { insaturados ômega-3 aumentaram. Já após } 6 \text { meses de intervenção nutricional, os } \\
\text { pacientes não apresentaram alterações significativas nos sinais neurológicos. } \\
\text { Dessa forma, diante os resultados apresentados observa-se que a intervenção } \\
\text { nutricional pode melhorar o status físico e inflamatório de pacientes com EM, visto } \\
\text { principalmente nos primeiros } 3 \text { meses de uso. }\end{array}$ \\
\hline $\begin{array}{l}\text { Immune regulatory } \\
\text { effects of high dose } \\
\text { vitamin } \quad \text { D3 } \\
\text { supplementation in a }\end{array}$ & 2016 & Anne-Hilde Muris & $\begin{array}{l}\text { Após análise do estudo proposto através da suplementação da vitamina } \mathrm{D} \\
\text { promovida altas doses em pacientes com EM, apresentou resultados sem efeitos } \\
\text { benéficos substanciais no sistema Imunológico. Todavia, os resultados mostram } \\
\text { que a suplementação de Vitamina D promove a prevenção da deterioração ou }\end{array}$ \\
\hline
\end{tabular}




\begin{tabular}{|c|c|c|c|}
\hline \begin{tabular}{lr}
\multicolumn{2}{l}{ randomized } \\
controlled trial in \\
relapsing & remitting \\
multiple & sclerosis \\
patients receiving \\
IFN $\beta ; \quad r \quad$ the \\
SOLARIUM study
\end{tabular} & & & $\begin{array}{l}\text { desequilíbrio imunológico causado pela EM. Podendo dessa forma, concluir que } \\
\text { essa suplementação de vitamina D deve ser mais eficaz nas fases inicial do curso } \\
\text { da doença. }\end{array}$ \\
\hline $\begin{array}{lrr}\text { Percepção } & & \text { do } \\
\text { paciente } & & \text { com } \\
\text { esclerose } & \text { múltipla } \\
\text { acerca do uso } & \text { de } \\
\text { vitamina D } & \text { na } \\
\text { terapia clínica } & \end{array}$ & 2017 & $\begin{array}{lr}\text { Cássio } & \text { Fernando } \\
\text { Alves } & \text { Reis } \\
\text { Fernanda } & \text { Pereira } \\
\text { Guimarães }\end{array}$ & $\begin{array}{l}\text { Em um estudo da Finlândia onde foram selecionados pacientes que estavam em } \\
\text { surto de EM e outros que estavam na fase de remissão, foi possível verificar que } \\
\text { as concentrações de Vitamina D eram menores nos pacientes em surtos e que as } \\
\text { recaídas diminuíram em } 34 \% \text { na proporção que } 10 \text { ng/dl foram aumentados nas } \\
\text { concentrações da vitamina D. Além disso, antes da suplementação foi evidenciado } \\
\text { que } 56,4 \% \text { dos entrevistados tinham surtos anuais de } 1 \text { a } 2 \text { vezes e que após a } \\
\text { suplementação } 87,2 \% \text { dos entrevistados tiveram redução para zero surtos ao ano, } \\
\text { evidenciando melhora das manifestações clinicas. Vale ressaltar também que no } \\
\text { estudo foi informado pelos entrevistados que antes da suplementação de } \\
\text { colecalciferol } 17 \% \text { alegaram que havia melhora independente da Vitamina D, } \\
52,1 \% \text { afirmaram que apresentava piora e } 30,9 \% \text { relataram que o quadro era } \\
\text { estável. Com a suplementação de Vitamina D } 74,5 \% \text { dos pacientes alegaram } \\
\text { melhorar o quadro clínico, } 2,1 \% \text { pioraram e } 23,4 \% \text { permaneceram estáveis, } \\
\text { constatando, assim a qualidade de vida e bem estar da maioria dos pacientes. }\end{array}$ \\
\hline
\end{tabular}

Fonte: Autores (2021).

Neste presente estudo, são relatados resultados positivos e negativos sobre a relação da vitamina $\mathrm{D}$ em pacientes com esclerose múltipla. A suplementação de vitamina D mostrou efeitos positivos em 7 do total de artigos selecionados, apresentando seus benefícios por meio dos efeitos anti-inflamatórios na redução de células TCD-4 com função imunomoduladora e antioxidante. Além disso foi constatado redução de recidiva e melhora do bem estar do paciente. Tais artigos foram embasados em referências de 2019 e 2021 que corroboram com a evidência de que a vitamina D é eficaz. Na evidência da Revista MedScape discutida no $35^{\circ}$ congresso do European Committee for Treatment and Research in Multiple Sclerosis (ECTRIMS 2019), foi evidenciada a favor do uso da suplementação de vitamina D em indivíduos com esclerose múltipla pois ocasionam diminuição do risco de novas lesões ativas além de reduzir a progressão de incapacidade e evidenciar menor perda de volume cerebral. Ademais, os mais recentes resultados são os que evidenciam a eficácia da vitamina D sendo visto nos artigos de 2021 "Suplementação da vitamina D por longo período demonstra efeito protetor contra a recidiva da esclerose múltipla: revisão sistemática" demonstra através dos estudos que a dosagem diária de 10400 UI de colecaciferol por dia quando usadas por longos períodos, levam uma diminuição proporcional de células T CD41IL-171 e aumenta a quantidade da forma metabolicamente ativa da Vitamina D no plasma, e consequentemente, atua como fator de proteção contra a EM. No artigo "Revisão sistemática sobre a influência da vitamina d na esclerose múltipla e os prós e contras na suplementação oral” evidencia os níveis séricos de 25OHD $<50 \mathrm{~mol} / \mathrm{L}$ são considerados predisponentes para o desencadear da patologia, e as recaídas em pacientes com EM são menores em locais com suplementação oral. Por fim, foram analisados 2 artigos com efeitos negativo, que não mostram evidências cientificas suficientes que comprove a eficácia do uso da vitamina D no tratamento da esclerose múltipla.

\section{Conclusão}

Através do estudo desse trabalho foi possível concluir a eficiência da vitamina D como agente anti-inflamatório, antioxidante e imunomodulador com sua ação aliada ao sistema imunológico frente à esclerose múltipla. A combinação das respostas imunes inatas e adquiridas são potencializadas através da suplementação de vitamina D no organismo em altas doses, que ocasionam a diminuição do FNT e linfócitos T, o aumento de células anti-inflamatórias e inibição das citocinas pró inflamatórias. Ademais, auxiliam na maturação das células de defesa, diminuem as APC's suprimindo apresentação de antígenos. 
aumento a tolerância imune através da diferenciação dos linfócitos TH2 e participam na transcrição de AMPs. Justificando assim o uso de vitamina D, uma vez que sua suplementação está diretamente ligada a queda de casos de EM. Apesar de seu uso vir apresentando bons resultados diante o tratamento e diminuição de recidivas ainda não há um consentimento oficial no Brasil sobre a utilização da vitamina $\mathrm{D}$, sendo necessário também a unanimidade entre as doses administradas. Futuros estudos podem nos ajudar a entender melhor a relação entre vitamina D e a Esclerose Múltipla, sua relevância e eficácia no tratamento da Esclerose múltipla e sua funcionalidade na imunologia combatendo doenças autoimunes.

\section{Referências}

ABEM - Associação Brasileira de Esclerose Múltipla. Diagnóstico. 2016. <http://abem.org.br/esclerose/diagnostico/>.

Alves, B., Angeloni, R., Azzalis, L., Pereira, E., Perazzo, F., Rosa, P. C.\& Fonseca, F. (2014). Esclerose múltipla: revisão dos principais tratamentos da doença. Saúde e meio ambiente: Revista interdisciplinar, 3(2), 19-34.

Amado, E. T., Renatha, E. G. E. A., \& Claudia, C. C. (2015). Vitamina D associada ao sistema imunológico. Anais do EVINCI-UniBrasil, 1(4), 201-4.

Aranow, C. (2011). Vitamina D e o sistema imunológico. Journal of investigative medicine, 59 (6), 881-6.

Araújo, A. P. M., Lucena, I. G., Santos, J. L. V., Sousa, M. N. A. \& Lima, U. M. J. (2018). Uso de Vitamina D para prematuros. Journal of Medicine and Health Promotion, 3(1),956-964

Bertotti, A. P., Lenzi, M. C. R., \& Portes, J. R. M. (2011). O portador de Esclerose Múltipla e suas formas de enfrentamento frente à doença. Barbaroi, (34), 101-24.

Bhargava, P., Steele, S. U., Waubant, E., Revirajan, N. R., Marcus, J., Dembele, M. \& Mowry, E. M. (2016). Multiple sclerosis patients have a diminished serologic response to vitamin D supplementation compared to healthy controls. Multiple Sclerosis Journal, 22(6), 753-760.

Biasebetti, M. D. B. C., Rodrigues, I. D., \& Mazur, C. E. (2018). Relação do consumo de vitaminas e minerais com o sistema imunitário: uma breve revisão. Visão Acadêmica, 19(1).

Brito, B. B. O., Soares, R. X., Alexandre, T. A., Sousa, M. N. A., Júnior, U. M.L. (2017). Vitamina D: Relação com a imunidade e prevalência de doenças. Journal of Medicine and Health Promotion. 2 (2),598-608.

Brum, D.G, Comini-Frota, E.R, Vasconcelos, C.C.F, \& Dias-Tosta, E. (2014). Suplementação e uso terapêutico de vitamina D nos pacientes com esclerose múltipla: Consenso do Departamento Científico de Neuroimunologia da Academia Brasileira de Neurologia. Arquivos de Neuro-Psiquiatria, 72 ,152-6.

Campos, D. S. C. (2015). Esclerose múltipla: fatores nutricionais e fisiopatologia (Doctoral dissertation).

Castro, L. C. G. D. (2011). O sistema endocrinológico vitamina D. Arquivos Brasileiros de Endocrinologia \& Metabologia, 55, 566-75.

Catarino, AM, Claro, C., \& Viana, I. (2016). Vitamina D - Perspectivas Atuais. Jornal da Sociedade Portuguesa de Dermatologia e Venereologia, 74 (4), $345-$ 53.

Errante, P. R., Ferraz, R. R. N., \& Rodrigues, F. S. M. (2016). Esclerose múltipla: tratamento farmacológico e revisão de literatura. UNILUS Ensino e Pesquisa, 13(30), 105-117.

Galvão, L. O., Galvão, M. F., Reis, C. M. S., Batista, C. M. A., \& Casulari, L. A. (2013). Considerações atuais sobre a vitamina D. Brasília Med, 50(4), 324332.

Gomes, F. M., Almeida, J. F., \& Teles, M. J. (2013). Vitamina D-Uma Promessa na Esclerose Múltipla Vitamin D-a Multiple Sclerosis’ Promise. Sinapse, 9.

Laursen, J. H., Søndergaard, H. B., Sørensen, P. S., Sellebjerg, F., \& Oturai, A. B. (2016). A suplementação de vitamina D reduz a taxa de recaída em pacientes com esclerose múltipla recorrente-remitente tratados com natalizumabe. Esclerose múltipla e doenças relacionadas,10, $169-73$.

Maeda, S. S., Borba, V. Z., Camargo, M. B. R., Silva, D. M. W., Borges, J. L. C., Bandeira, F., \& Lazaretti-Castro, M. (2014). Recomendações da Sociedade Brasileira de Endocrinologia e Metabologia (SBEM) para o diagnóstico e tratamento da hipovitaminose D. Arquivos Brasileiros de Endocrinologia \& Metabologia, 58, 411-33.

Marques, C. D. L., Dantas, A. T., Fragoso, T. S., \& Duarte, Â. L. B. P. (2010). A importância dos níveis de vitamina D nas doenças autoimunes. Revista Brasileira de Reumatologia, 50, 67-80.

Muris, AH, Smolders, J., Rolf, L., Thewissen, M., Hupperts, R., Damoiseaux, J., \& Solarium Study Group. (2016). Efeitos imunorregulatórios da suplementação de vitamina D3 em altas doses em um estudo controlado randomizado em pacientes com esclerose múltipla recorrente-remitente recebendo IFN $\beta$; o estudo Solarium. Journal of neuroimmunology. 300, 47-56.

Reis, C. F. A., \& Guimarães, F. P. (2017). Percepção do Paciente com Esclerose Múltipla acerca do uso de Vitamina D na Terapia Clínica. Revista Brasileira de Ciências da Vida, 5(1).

Riccio, P., Rossano, R., Larocca, M., Trotta, V., Mennella, I., Vitaglione, P., \& Coniglio, MG (2016). Intervenção nutricional antiinflamatória em pacientes com esclerose múltipla recorrente-remitente e primária progressiva: Um estudo piloto. Experimental Biology and Medicine, 241 (6), 620-35. 
Research, Society and Development, v. 10, n. 15, e246101522575, 2021

(CC BY 4.0) | ISSN 2525-3409 | DOI: http://dx.doi.org/10.33448/rsd-v10i15.22575

Silva, V. M., \& Silva, D. F. (2014). Esclerose múltipla: imunopatologia, diagnóstico e tratamento-artigo de revisão. Interfaces Científicas-Saúde e Ambiente, 2(3), 81-90.

Siqueira, M. K., \& Pires, L. (2016). Processo epigenéticos envolvidos na fisiopatologia da esclerose múltipla com ênfase na função dos miRNAs. Semina: Ciências Biológicas e da Saúde, 37(2), 125-136.

Sotirchos, ES, Bhargava, P., Eckstein, C., Van Haren, K., Baynes, M., Ntranos, A., \& Calabresi, P. A. (2016). Safety and immunologic effects of high-vs lowdose cholecalciferol in multiple sclerosis. Neurology, 86 (4), 382-390.

Souza, A. W. S. D., Mesquita Júnior, D., Araújo, J. A. P., Catelan, T. T. T., Cruvinel, W. D. M., Andrade, L. E. C., \& Silva, N. P. D. (2010). Sistema imunitário: parte III. O delicado equilíbrio do sistema imunológico entre os pólos de tolerância e autoimunidade. Revista Brasileira de Reumatologia, 50, 665-679.

Souza, M. T. D., Silva, M. D. D., \& Carvalho, R. D. (2010). Revisão integrativa: o que é e como fazer. Einstein (São Paulo), 8, 102-106. 